\title{
Protective effects of allicin against ischemic stroke in a rat model of middle cerebral artery occlusion
}

\author{
BENPING ZHANG ${ }^{1}$, FENG LI ${ }^{1}$, WEIJIANG ZHAO ${ }^{2}$, JIEBING LI ${ }^{3}$, QINGSONG LI ${ }^{1}$ and WEIZHI WANG ${ }^{1}$ \\ ${ }^{1}$ Department of Neurology, The Second Affiliated Hospital, Harbin Medical University, Harbin, Heilongjiang 150086; \\ ${ }^{2}$ Center for Neuroscience, Medical College of Shantou University, Shantou, Guangdong 515041; ${ }^{3}$ Department of Ultrasound, \\ The Affiliated Tumor Hospital, Harbin Medical University, Harbin, Heilongjiang 150086, P.R. China
}

Received August 28, 2014; Accepted May 13, 2015

DOI: $10.3892 / \mathrm{mmr} .2015 .3883$

\begin{abstract}
Allicin, a molecule predominantly responsible for the pungent odor and the antibiotic function of garlic, exhibits various pharmacological activities and has been suggested to be beneficial in the treatment of various disorders. The present study aimed to elucidate the effect of allicin in cerebral ischemia/reperfusion (I/R) injury in rats. Rats were subjected to $1.5 \mathrm{~h}$ of transient middle cerebral artery occlusion (MCAO), followed by $24 \mathrm{~h}$ of reperfusion. Rats were randomly assigned to the sham surgery group, the MCAO group and the $\mathrm{MCAO}+$ allicin group. Neurological score, cerebral infarct size, brain water content, neuronal apoptosis, serum tumor necrosis factor (TNF)- $\alpha$ and myeloperoxidase (MPO) activity were measured. The results suggested that allicin reduced cerebral infarction area, brain water content, neuronal apoptosis, TNF- $\alpha$ levels and MPO activity in the serum. The results of the present study indicated that allicin protects the brain from cerebral I/R injury, which may be ascribed to its anti-apoptotic and anti-inflammatory effects.
\end{abstract}

\section{Introduction}

Acute ischemic stroke has high rates of mortality and morbidity and is a major cause of mortality globally (1). Ischemic stroke accounts for almost $80 \%$ of all types of stroke, usually caused by a thrombotic or embolic occlusion of the middle cerebral artery or its branches (2). This occlusion triggers brain injury via a complex series of pathophysiological processes, resulting in neuronal death or neuronal apoptosis and subsequent development of neurological disorders. There is an urgent requirement for the development of neuroprotective therapies for acute cerebral ischemia, which salvage the ischemic

Correspondence to: Dr Weizhi Wang, Department of Neurology, The Second Affiliated Hospital, Harbin Medical University, 248 Xuefu Road, Harbin, Heilongjiang 150086, P.R. China E-mail: wangweizhi2009@126.com

Key words: allicin, cerebral ischemic stroke, middle cerebral artery occlusion, tumor necrosis factor- $\alpha$, myeloperoxidase penumbra. This strategy is reflected by the increasing use of acute re-canalization therapies, including thrombolysis (3) and the mechanical removal of clots (4). Various neuroprotective compounds have been developed to treat ischemic damage (5); however, satisfactory drug treatments in clinical practice are limited.

Allium sativum, commonly known as garlic, has been widely utilized globally as a condiment. Previous studies have suggested possible medicinal applications and health benefits of garlic $(6,7)$. In addition, garlic has been used for a long time in Traditional Chinese Medicine in the treatment of various diseases (8).

Allicin (diallyl thiosulfinate), known as one of the most active components of garlic, is responsible for the typical smell and numerous beneficial functions of garlic (9). Previous studies have demonstrated that allicin possesses a wide spectrum of pharmacological effects, including anti-inflammatory, anti-fungal, anti-oxidant and anti-tumoral activities $(10,11)$. Allicin has also been reported to exhibit protective effects on the brain. It has been suggested that allicin mitigates traumatic brain injury (12). However, whether allicin protects the brain from ischemic injury remains to be elucidated. Therefore, the present study aimed to elucidate the effect of allicin and its underlying mechanisms of action in a rat model middle cerebral artery occlusion (MCAO).

\section{Materials and methods}

Reagents. Allicin (purity, >98\%) was purchased from the National Institute for the Control of Pharmaceutical and Biological Products (Beijing, China). A tumor necrosis factor (TNF)- $\alpha$ ELISA kit was purchased from Beyotime Institute of Biotechnology (Shanghai, China). A myeloperoxidase (MPO) assay kit was purchased from Nanjing Jiancheng Bioengineering Institute (Nanjing, China). 2,3,5-triphenyltetrazolium chloride (TTC) was purchased from Sigma-Aldrich (St. Louis, MO, USA).

Animals. A total of 60 adult male Sprague-Dawley rats (250-300 g; 6-8 weeks-old) were purchased from the Experimental Animal Center of Harbin Medical University (Harbin, China). All animals were maintained under a 12-h light/dark regime with ad libitum access to food and water. 
The rats were housed at $22^{\circ} \mathrm{C}$ with $50 \%$ relative humidity. The animals were maintained in accordance with the Guide for the Care and Use of Laboratory Animals published by the United States' National Institutes of Health (publication no. 85-23, revised 1996; Bethesda, MD, USA). The protocol was approved by the Committee of Experimental Animals of Harbin Medical University (Harbin, China).

Preparation of MCAO model. Rats were randomly assigned to three groups: Sham-surgery group $(n=20)$, MCAO group $(n=20)$ and MCAO + allicin group $(n=20)$. The rats were anesthetized intraperitoneally (i.p.) with chloral hydrate $(350 \mathrm{mg} / \mathrm{kg}$; Beyotime Institute of Biotechnology). Subsequently, the rat was fixed in a supine position and a midline skin incision was made to expose the right common carotid artery, the internal carotid artery (ICA) and the external carotid artery (ECA). The MCAO was performed as described previously (13). Briefly, a 4-0 nylon monofilament (Sunbio Biotech, Beijing, China) with a head-rounded tip was inserted into the ECA and gently advanced into the ICA for $20 \mathrm{~mm}$ to obstruct the blood flow of the right middle cerebral artery. After a 90-minute occlusion, the filament was withdrawn, allowing for the reperfusion for $24 \mathrm{~h}$. The rectal temperature of the rat was maintained at $37^{\circ} \mathrm{C} \pm 0.5^{\circ} \mathrm{C}$ using a heating pad. Rats in the sham group were subjected to the same procedure, with the exception of the MCAO. Allicin was administered at a dose of $50 \mathrm{mg} / \mathrm{kg}$ i.p. $3 \mathrm{~h}$ after reperfusion daily for five consecutive days. Allicin was dissolved in 2\% dimethyl sulfoxide (DMSO; Sigma-Aldrich). Rats in the sham and MCAO groups were injected with an equal volume of DMSO.

Neurological score. The neurological score was assessed by an observer blinded to the animal groups after $24 \mathrm{~h}$ reperfusion according to previously described methods (14). The neurological deficits were evaluated on a five-point scale as follows: No neurological deficits $=0$; failure to fully extend left paw $=1$; circling to the left $=2$; falling to the left $=3$; no spontaneous walking and depressed levels of consciousness $=4$.

Evaluation of brain water content. At $24 \mathrm{~h}$ after MCAO, the brain water content was detected according to a previously described method (15). The rats were sacrificed by deep anesthesia with chloral hydrate $(350 \mathrm{mg} / \mathrm{kg}$, i.p.) and brains were immediately removed and placed on a frozen plate. Tissue samples were collected from infarct areas of ischemic rats and from the corresponding areas in the sham-surgery rats. The samples were weighed to determine the wet weight. Subsequently, samples were dried in a desiccating oven at $110^{\circ} \mathrm{C}$ for $24 \mathrm{~h}$ and then weighed to determine the dry weight. The brain water content was calculated using the following formula: brain water content $(\%)=$ (wet weight - dry weight) x100\%/wet weight.

Evaluation of infarct size. The animals were sacrificed $24 \mathrm{~h}$ after reperfusion ( $n=6$ in each group) by deep anesthesia with chloral hydrate $(350 \mathrm{mg} / \mathrm{kg}$, i.p.). The brains were rapidly removed and cut into five coronal sections $(2 \mathrm{~mm})$. The sections were incubated in a solution of $2 \% \mathrm{TTC}$ at $37^{\circ} \mathrm{C}$ for $30 \mathrm{~min}$. The infarct sizes were measured using Image J software version 1.6 (National Institutes of Health).
Immunohistochemistry. At $24 \mathrm{~h}$ after reperfusion, the rats were anesthetized and transcardially perfused with saline, followed by $4 \%$ paraformaldehyde (Beijing Solarbio Science \& Technology Co., Ltd., Beijing, China) in phosphate-buffered saline. Subsequently, the samples were dehydrated in a graded series of ethanol and embedded in paraffin (Leica Biosystems, Wetzlar, Germany). Following epitope retrieval at $120^{\circ} \mathrm{C}$ for $5-10 \mathrm{~min}$ using citrate buffer (10 mM citric acid, $0.05 \%$ Tween 20; $\mathrm{pH}$ 6.0), the sections were incubated with $3 \% \mathrm{H}_{2} \mathrm{O}_{2}$ for $15 \mathrm{~min}$ and then with $5 \%$ bovine serum albumin (Beyotime Institute of Biotechnology) for $1 \mathrm{~h}$. The sections were then incubated with primary mouse monoclonal neuronal nuclei (NeuN) antibody (1:100; cat. no. MAB377; EMD Millipore, Billerica, MD, USA) overnight at $4^{\circ} \mathrm{C}$, followed by incubation with goat anti-mouse secondary antibody (1:1,000; P044701; Dako, Carpinteria, CA, USA) for $30 \mathrm{~min}$ at $37^{\circ} \mathrm{C}$. The immunoreactivity was visualized using a Dako Envision system-horseradish peroxidase kit (Dako). Finally, counterstaining was performed using hematoxylin (Beyotime Institute of Biotechnology). NeuN-positive staining in the cortex was identified by the presence of deep brown staining of cells specifically localized to the nucleus (TI-S; Nikon Corporation, Tokyo, Japan). The images were analyzed using the Image-Pro plus 6.0 image analysis system (Media Cybernetics, Inc., Rockville, MD, USA).

Western blot analysis. Total proteins of the cortex were extracted and quantified according to the bicinchoninic acid protein assay kit (Beyotime Institute of Biotechnology). The protein samples were subjected to $8 \%$ SDS-PAGE (Beijing Solarbio Science \& Technology Co., Ltd.) followed by electrotransfer onto polyvinylidene difluoride membranes (Millipore, Boston, MA, USA). Subsequently, the membranes were blocked with $5 \%$ non-fat milk for $2 \mathrm{~h}$ at room temperature. The membranes were then incubated overnight at $4^{\circ} \mathrm{C}$ with the rabbit anti-cleaved caspase-3 polyclonal antibody (1:1,000; cat. no. 9661 ; Cell Signaling Technology, Inc., Danvers, MA, USA) and with rabbit anti- $\beta$-actin polyclonal antibody (1:3,000; sc-7210; Santa Cruz Biotechnology, Inc., Dallas, TX, USA), followed by three washes with Tris-buffered saline with Tween-20 (TBST; Beijing Solarbio Science \& Technology Co., Ltd.). Subsequently, the membranes were incubated with the secondary antibody (1:5,000; Sc-2004; goat anti-rabbit immunoglobulin G-horseradish peroxidase; Santa Cruz Biotechnology, Inc.) for $2 \mathrm{~h}$ at room temperature, followed by three washes with TBST. Finally, the bands were detected via chemiluminescence with the ECL Plus western blotting detection kit (EMD Millipore). Blots were analyzed using the Bio-Rad Imaging software (Bio-Rad Laboratories, Inc., Hercules, CA, USA) and Quantity One software package version 4.6.2 (Bio-Rad Laboratories).

Measurement of serum TNF- $\alpha$. At $24 \mathrm{~h}$ after the MCAO, blood samples $(1 \mathrm{ml})$ were harvested from the femoral vein. Following centrifugation at $1,000 \mathrm{x}$ g for $15 \mathrm{~min}$, the supernatant was collected and stored at $-80^{\circ} \mathrm{C}$. Serum TNF- $\alpha$ was assayed using a TNF- $\alpha$ ELISA kit according to the manufacturer's instructions.

Evaluation of MPO. Following reperfusion, brain tissue from the penumbral cortex was immediately harvested and stored at $-80^{\circ} \mathrm{C}$. The brain tissue was homogenized in ice-cold 


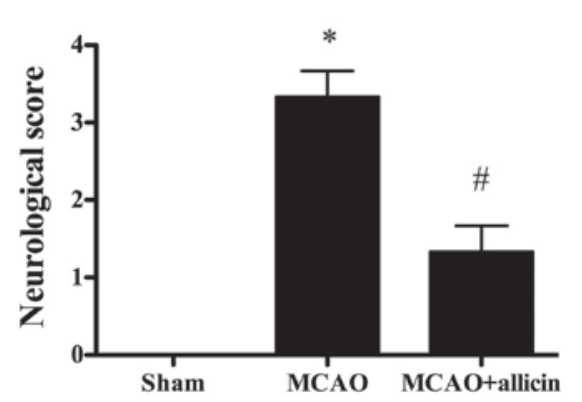

Figure 1. Effect of allicin on neurological deficits. Allicin significantly improved neurological recovery compared with that in the MCAO group at $24 \mathrm{~h}$ after reperfusion. Values are expressed as the mean \pm standard deviation $(\mathrm{n}=6) .{ }^{*} \mathrm{P}<0.05$ versus the sham group; ${ }^{*} \mathrm{P}<0.05$ versus the MCAO group. $\mathrm{MCAO}$, middle cerebral artery occlusion.

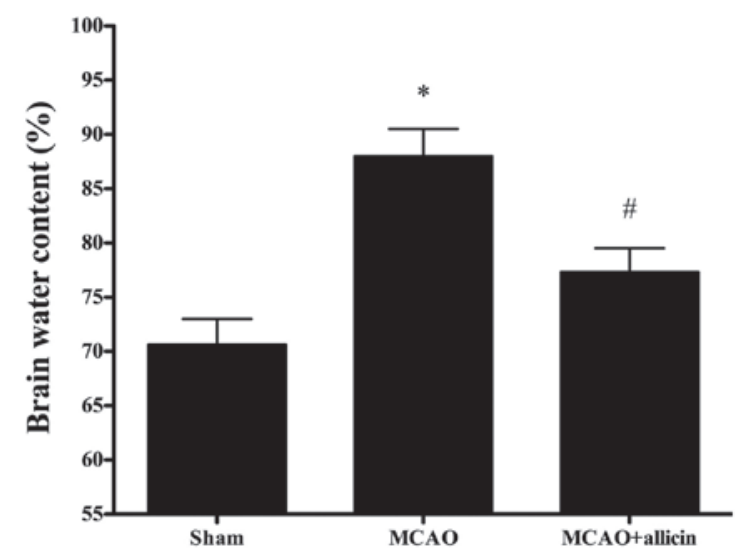

Figure 2. Allicin reduces brain edema at $24 \mathrm{~h}$ after MCAO in rats. The brain water content of rats is shown. Values are expressed as the mean \pm standard deviation $(n=6) .{ }^{*} \mathrm{P}<0.05$, versus the sham group; ${ }^{~} \mathrm{P}<0.05$, versus the MCAO group. MCAO, middle cerebral artery occlusion.

phosphate-buffered saline. Subsequently, the homogenate was centrifuged at $1,000 \mathrm{x}$ g for $15 \mathrm{~min}$. MPO was detected using an MPO kit according to manufacturer's instructions (Nanjing Jiancheng Bioengineering Institute).

Statistical analysis. Values are expressed as the mean \pm standard deviation. Statistical analyses were performed using the SPSS 16.0 software package (SPSS, Inc., Chicago, IL, USA). Data analysis was performed by one-way analysis of variance, followed by a least significant difference-t-test for inter-group comparisons. $\mathrm{P}<0.05$ was considered to indicate a statistically significant difference.

\section{Results}

Allicin ameliorates neurological deficits following MCAO. As shown in Fig. 1, the rats in the sham group did not exhibit any neurological deficits, while the MCAO rats exhibited severe neurological deficits according the neurological scoring system. Allicin treatment markedly ameliorated the neurological deficits compared with those in the MCAO group $(\mathrm{P}<0.05)$.

Allicin decreases brain edema following MCAO. At $24 \mathrm{~h}$ after MCAO, the brain water content of the MCAO rats was markedly higher than that of the sham-surgery rats. Treatment with allicin
A
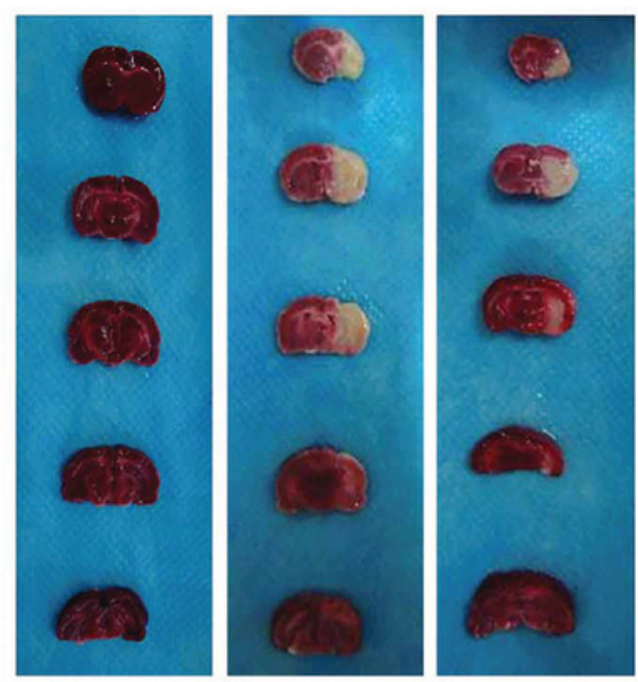

B

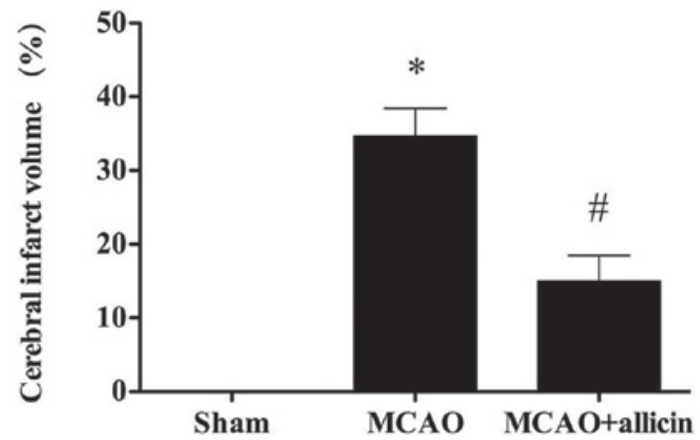

Figure 3. Allicin reduces the infarct volume in an MCAO model (A) Representative images of 2,3,5-triphenyltetrazolium chloride-stained cerebral sections in each group. The normal tissue was stained dark red, while the infarct tissue was white. (B) Quantitative analysis of the infarct volume. Values are expressed as the mean \pm standard deviation $(n=6)$. $P<0.05$, versus the sham group; ${ }^{\#} \mathrm{P}<0.05$, versus the $\mathrm{MCAO}$ group. $\mathrm{MCAO}$, middle cerebral artery occlusion.

significantly decreased brain edema compared with those in the MCAO group ( $\mathrm{P}<0.05$; Fig. 2).

Allicin reduces the brain infarct volume following MCAO. The rats in the sham group did not exhibit any infarct area in the brain, while in the MCAO group, a clear infarction was observed. Following treatment with allicin, the infarct volume in the MCAO + allicin group was significantly lower compared with that in the MCAO group $(\mathrm{P}<0.05$; Fig. 3$)$.

Allicin rescues neuronal survival following MCAO. Compared with the sham group, the number of NeuN-positive neurons was significantly decreased in the MCAO group. The decrease of NeuN-positive neurons was attenuated by allicin treatment $(\mathrm{P}<0.05$; Fig. 4).

Allicin inhibits cerebral ischemia-induced neuronal apoptosis. To identify whether the neuroprotective effect of allicin was associated with its anti-apoptotic activity, the levels of cleaved caspase- 3 were assessed. Compared with those in the sham group, the levels of cleaved caspase-3 were significantly increased in the MCAO group. Of note, allicin markedly decreased the levels of cleaved caspase-3 (P<0.05; Fig. 5). 

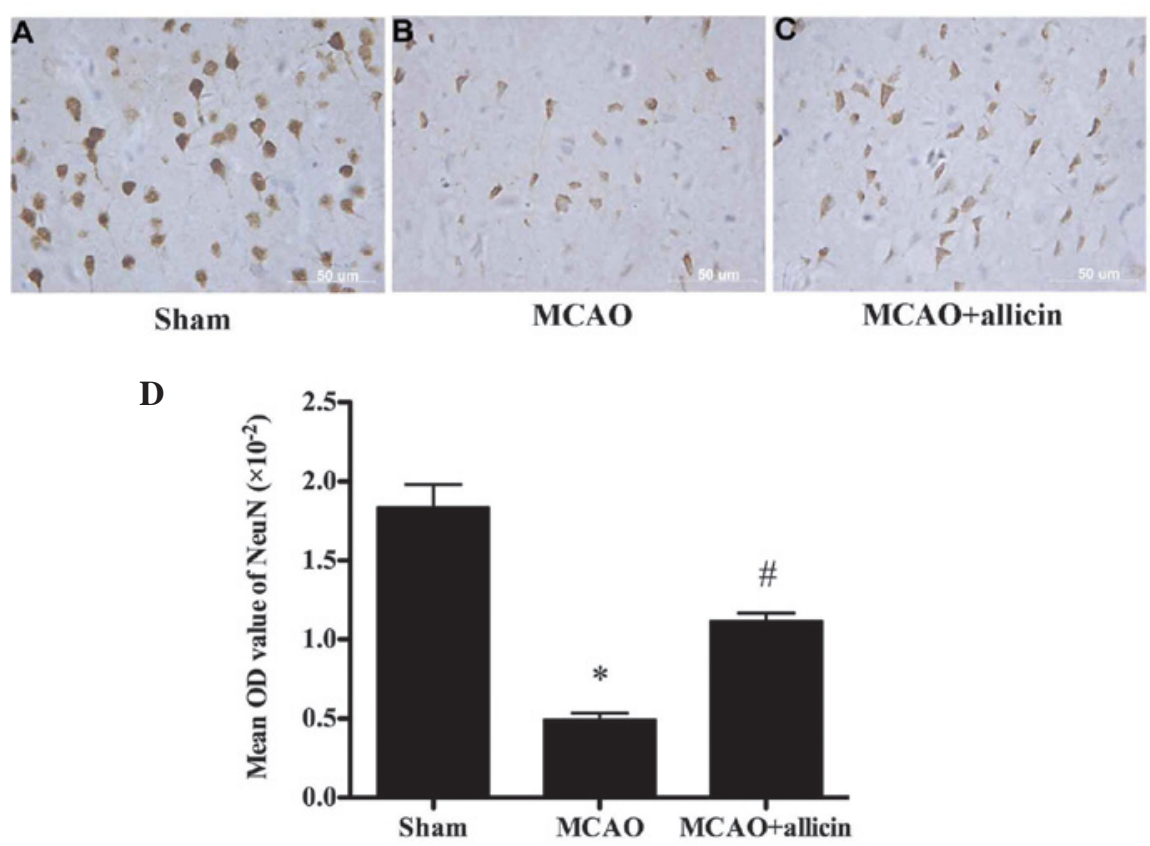

Figure 4. Allicin decreases neuronal apoptosis during MCAO. (A-C) NeuN (neuronal marker) immunohistochemical staining in the sham, MCAO and MCAO + allicin groups (scale bar, $50 \mu \mathrm{m}$ ). (D) Quantitative analysis of the mean optical density values for NeuN. Values are expressed as the mean \pm standard deviation ( $\mathrm{n}=6$ ). ${ }^{\mathrm{P}} \mathrm{P}<0.05$, vs. sham group; ${ }^{\mathrm{P}} \mathrm{P}<0.05$, vs. MCAO group. MCAO, middle cerebral artery occlusion; NeuN, neuronal nuclei; OD, optical density.

A

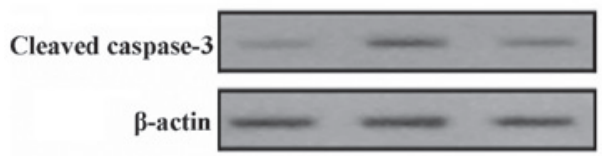

B

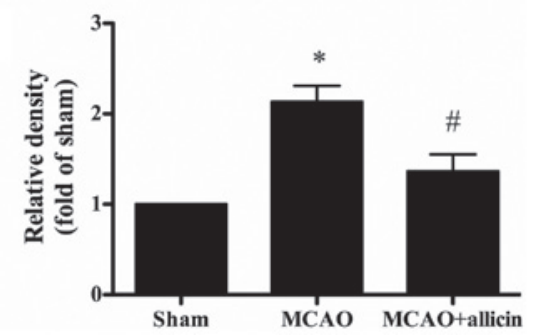

Figure 5. Allicin attenuates neuronal apoptosis induced by cerebral ischemia/reperfusion injury. (A) Representative western blot showing the levels of cleaved caspase-3. (B) Relative density of cleaved caspase-3 (fold of sham) in the experimental groups. Values are expressed as the mean \pm standard deviation $(n=6) .{ }^{*} \mathrm{P}<0.05$, versus the sham group; ${ }^{~} \mathrm{P}<0.05$, versus the MCAO group. MCAO, middle cerebral artery occlusion.

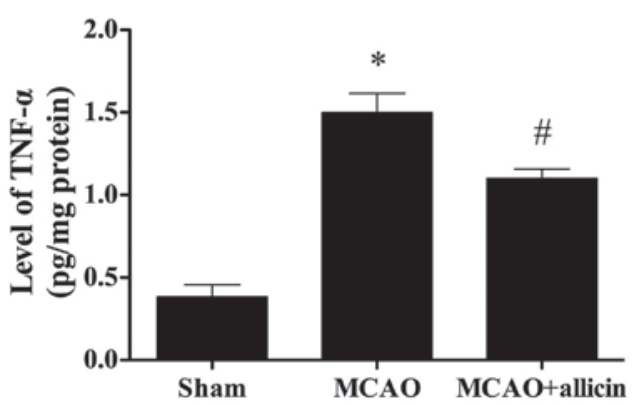

Figure 6. Serum levels of TNF- $\alpha$ in each group. Compared with the MCAO group, allicin significantly reduced TNF- $\alpha$ levels in the MCAO + allicin group. Values are expressed as the mean \pm standard deviation $(n=6) .{ }^{*} \mathrm{P}<0.05$, versus the sham group; ${ }^{\#} \mathrm{P}<0.05$, versus the MCAO group. MCAO, middle cerebral artery occlusion; TNF, tumor necrosis factor.

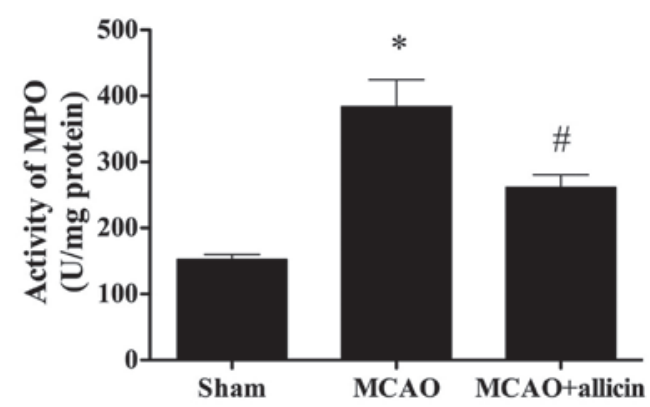

Figure 7. Comparison of MPO activity in each group. Compared with the MCAO group, the MPO activity in the MCAO + allicin group was markedly decreased. Values are expressed as the mean \pm standard deviation $(n=6)$. ${ }^{*} \mathrm{P}<0.05$, versus the sham group; ${ }^{\#} \mathrm{P}<0.05$, versus the MCAO group. MCAO, middle cerebral artery occlusion; MPO, myeloperoxidase.

Allicin attenuates increases in serum levels of TNF- $\alpha$ following $M C A O$. A cerebral ichemia/reperfusion (I/R) injury causes the production of TNF- $\alpha$ (16). Thus, the levels of serum TNF- $\alpha$ were assessed. Fig. 6 shows that, compared with the MCAO group, allicin significantly decreased the serum levels of TNF- $\alpha$ $(\mathrm{P}<0.05)$.

Allicin attenuates increases in MPO activity following MCAO. As indicated in Fig. 7, the MPO activity in the sham group was at a normal level, while that in the MCAO group was markedly elevated $(\mathrm{P}<0.05)$. Of note, treatment with allicin significantly lowered cerebral MPO activity $(\mathrm{P}<0.05)$.

\section{Discussion}

In the present study, it was demonstrated that allicin exerted a neuroprotective effect against cerebral I/R injury. Treatment with allicin significantly ameliorated neurological deficits, 
decreased the cerebral infarct area, as well as attenuated neuronal death, apoptosis and inflammation. Therefore, the results of the present study suggested that allicin may be of therapeutic value in the treatment of ischemic stroke.

In the signaling pathways involved in apoptosis, ischemia induces the translocation of B-cell lymphoma 2-associated $\mathrm{X}$ protein into the outer membrane of the mitochondria, leading to the release of cytochrome $\mathrm{C}$ from the mitochondria to the cytoplasm. This process causes the activation of pro-caspase-3 into active caspase-3 (cleaved caspase-3), a critical executioner of apoptosis (17), triggering apoptosis $(18,19)$.

Apoptosis, induced by cerebral I/R injury, is one of the major causes of cell death in the ischemic penumbra (20). The infarct size is determined by the number of apoptotic neurons in the penumbra (21). Therefore, inhibiting apoptosis in the ischemic penumbra may be a promising therapeutic target for mitigating cerebral infarct size following cerebral I/R injury. In the present study, the number of NeuN-positive cells in the MCAO group was significantly decreased, while it was markedly increased following allicin treatment. In addition, cleaved caspase-3 levels were increased in rats subjected to MCAO, which was attenuated by allicin treatment. Therefore, the results of the present study demonstrated that allicin has anti-apoptotic effects.

In addition, inflammation is critical in brain injury and infarction induced by cerebral ischemia. During inflammation, inflammatory cytokines and cells are of great significance. Inflammation-associated cytokines include proand anti-inflammatory cytokines according to their ability to activate or inhibit inflammation (22). Important pro-inflammatory cytokines, including TNF- $\alpha$, interleukin (IL)- $1 \beta$ and IL-6, are responsible for the initiation of inflammatory reactions and inducing the expression of other cytokines following I/R injury (23). In the present study, allicin markedly reduced TNF- $\alpha$ and MPO activity, indicating that allicin protects the brain from ischemia via an anti-inflammatory pathway.

In conclusion, the present study indicated that allicin has a protective effect against cerebral I/R injury in rats, which may be ascribed to its anti-inflammatory and anti-apoptotic properties. The findings of the present study provided further insight into the mechanism by which allicin exerts its neuroprotective effects and paved a way for the development of a novel therapeutic target in the clinical treatment of cerebral ischemic stroke.

\section{References}

1. Saito T, Nito C, Ueda M, et al: Continuous oral administration of atorvastatin ameliorates brain damage after transient focal ischemia in rats. Life Sci 94: 106-114, 2014.

2. Durukan A and Tatlisumak T: Acute ischemic stroke: Overview of major experimental rodent models, pathophysiology, and therapy of focal cerebral ischemia. Pharmacol Biochem Behav 87: 179-197, 2007.
3. Hacke W, Kaste M, Bluhmki E, et al; ECASS Investigators: Thrombolysis with alteplase 3 to 4.5 hours after acute ischemic stroke. N Engl J Med 359: 1317-1329, 2008.

4. Smith WS, Sung G, Saver J, et al; Multi MERCI Investigators: Mechanical thrombectomy for acute ischemic stroke: Final results of the Multi MERCI trial. Stroke 39: 1205-1212, 2008.

5. Katsura K, Suda S, Abe A, Kanamaru T, Toda Y and Katayama Y: Brain protection therapy in acute cerebral infarction. J Nippon Med Sch 79: 104-110, 2012

6. Asdaq SM: Antioxidant and hypolipidemic potential of aged garlic extract and its constituent, s-allyl cysteine, in rats. Evid Based Complement Alternat Med 2015: 328545, 2015.

7. Arreola R, Quintero-Fabián S, López-Roa RI, Flores-Gutiérrez EO, Reyes-Grajeda JP, Carrera-Quintanar L and Ortuño-Sahagún D: Immunomodulation and anti-inflammatory effects of garlic compounds. J Immunol Res 2015: 401630, 2015.

8. Ginter E and Simko V: Garlic (Allium sativum L.) and cardiovascular diseases. Bratisl Lek Listy 111: 452-456, 2010.

9. Lawson LD and Gardner CD: Composition, stability, and bioavailability of garlic products used in a clinical trial. J Agric Food Chem 53: 6254-6261, 2005.

10. Hunter R, Caira M and Stellenboom N: Thiolsulfinate allicin from garlic: Inspiration for a new antimicrobial agent. Ann NY Acad Sci 1056: 234-241, 2005.

11. Chan JY, Tsui HT, Chung IY, Chan RY, Kwan YW and Chan SW: Allicin protects rat cardiomyoblasts ( $\mathrm{H} 9 \mathrm{c} 2$ cells) from hydrogen peroxide-induced oxidative injury through inhibiting the generation of intracellular reactive oxygen species. Int J Food Sci Nutr 65: 868-873, 2014.

12. Zhou YF, Li WT, Han HC, et al: Allicin protects rat cortical neurons against mechanical trauma injury by regulating nitric oxide synthase pathways. Brain Res Bull 100: 14-21, 2014.

13. Ye R, Yang Q, Kong X, et al: Ginsenoside Rd attenuates early oxidative damage and sequential inflammatory response after transient focal ischemia in rats. Neurochem Int 58: 391-398, 2011.

14. Longa EZ, Weinstein PR, Carlson S and Cummins R: Reversible middle cerebral artery occlusion without craniectomy in rats. Stroke 20: 84-91, 1989.

15. Hatashita S, Hoff JT and Salamat SM: Ischemic brain edema and the osmotic gradient between blood and brain. J Cereb Blood Flow Metab 8: 552-559, 1988.

16. Saad MA, Abdelsalam RM, Kenawy SA and Attia AS: Montelukast, a cysteinyl leukotriene receptor-1 antagonist protects against hippocampal injury induced by transient global cerebral ischemia and reperfusion in rats. Neurochem Res 40: 139-150, 2015.

17. Fernandes-Alnemri T, Litwack G and Alnemri ES: CPP32, a novel human apoptotic protein with homology to Caenorhabditis elegans cell death protein Ced-3 and mammalian interleukin-1 beta-converting enzyme. J Biol Chem 269: 30761-30764, 1994.

18. Lee Y and Gustafsson AB: Role of apoptosis in cardiovascular disease. Apoptosis 14: 536-548, 2009.

19. Crow MT, Mani K, Nam YJ and Kitsis RN: The mitochondrial death pathway and cardiac myocyte apoptosis. Circ Res 95: 957-970, 2004.

20. Mattson MP, Duan W, Pedersen WA and Culmsee C: Neurodegenerative disorders and ischemic brain diseases. Apoptosis 6: 69-81, 2001.

21. Broughton BR, Reutens DC and Sobey CG: Apoptotic mechanisms after cerebral ischemia. Stroke 40: e331-e339, 2009.

22. Chen T, Liu W, Chao X, et al: Salvianolic acid B attenuates brain damage and inflammation after traumatic brain injury in mice. Brain Res Bull 84: 163-168, 2011.

23. Yasuda Y, Shimoda T, Uno K, et al: Temporal and sequential changes of glial cells and cytokine expression during neuronal degeneration after transient global ischemia in rats. J Neuroinflammation 8: 70, 2011. 